\title{
Electron quasi-itinerancy intertwined with quantum order by disorder in pyrochlore iridate magnetism
}

\author{
Gang Chen $\oplus^{1,2,3}$ and Xiaoqun Wang ${ }^{4,5}$ \\ ${ }^{1}$ Department of Physics and HKU-UCAS Joint Institute for Theoretical and Computational Physics at Hong Kong, \\ The University of Hong Kong, Hong Kong, China \\ ${ }^{2}$ State Key Laboratory of Surface Physics and Department of Physics, Institute of Nanoelectronics and Quantum Computing, \\ Fudan University, Shanghai 200433, China \\ ${ }^{3}$ Collaborative Innovation Center of Advanced Microstructures, Nanjing University, Nanjing 210093, China \\ ${ }^{4}$ School of Physics and Astronomy, Tsung-Dao Lee Institute, Shanghai Jiao Tong University, Shanghai 200240, China \\ ${ }^{5}$ Key Laboratory of Artificial Structures and Quantum Control, Ministry of Education, Shenyang National Laboratory for Materials Science, \\ Shenyang 110016, China
}

(Received 6 August 2020; accepted 4 November 2020; published 23 November 2020)

\begin{abstract}
We point out the emergence of magnetism from the interplay of electron quasi-itinerancy and quantum order by disorder in pyrochlore iridates. Like other Mott insulating iridates, $\mathrm{the}^{4+}{ }^{4+}$ ion in pyrochlore iridates develops an effective $J=1 / 2$ moment from the on-site spin-orbit coupling. We consider the generic symmetry-allowed exchange between these local moments on a pyrochlore lattice and obtain the mean-field phase diagram. Assuming the superexchange is mediated by direct and/or indirect electron hopping via intermediate oxygens, we derive the exchange interactions in the strong-coupling regime from the Hubbard model. This exchange has a degenerate classical ground-state manifold, and quantum fluctuation selects a noncoplanar ground state, known as quantum order by disorder. Extending to the intermediate-coupling regime, the same noncoplanar order is selected from the degenerate manifold by the kinetic energy, which is dubbed "electron quasi-itinerancy." We discuss the experimental relevance of our results and electron quasi-itinerancy among other iridates and $4 d / 5 d$ magnets.
\end{abstract}

DOI: 10.1103/PhysRevResearch.2.043273

\section{INTRODUCTION}

In recent years, there has been a lot of activity on the Ir-based transition-metal oxides. Due to the strong spin-orbit coupling (SOC) of its $5 d$ electrons, many novel phases, theoretical models, and experiments have been proposed and discovered in these Ir-based materials [1-4]. Among them, for example, a quantum spin liquid phase was proposed for an Ir-based hyperkagomé lattice in $\mathrm{Na}_{4} \mathrm{Ir}_{3} \mathrm{O}_{8}$ [5], and a ferromagnetic ground state with a large ferromagnetic moment was discovered in $\mathrm{Sr}_{2} \mathrm{IrO}_{4}$ with the $\mathrm{Ir}^{4+}$ ions forming a square lattice [6]. In these Mott insulating systems, the presence of strong SOC drastically changes the local spin physics. The local moment of the magnetic ion $\operatorname{Ir}^{4+}$ is an effective $J=1 / 2$ moment $[5,7,8]$ describing local spin-orbital doublets rather than the usual electron spin $S=1 / 2$ for systems with a weak SOC. The existence of local spin-orbital doublets has been detected by resonant $\mathrm{x}$-ray scattering experiments in $\mathrm{Sr}_{2} \mathrm{IrO}_{4}$ [9]. As a consequence, the nontrivial exchange interaction can arise due to the mixing of spin and orbitals $[5,10]$.

Published by the American Physical Society under the terms of the Creative Commons Attribution 4.0 International license. Further distribution of this work must maintain attribution to the author(s) and the published article's title, journal citation, and DOI.
Even though the superexchange interaction between the Ir local moments is often used to describe most iridates, most well-known Mott insulating iridates are actually weak Mott insulators with quasi-itinerant $5 d$ electrons and small charge gaps. This weak Mott insulating nature was not really emphasized in the literature, and we think this may be important in understanding some of the physical properties of iridates and the related materials. What is electron quasi-itinerancy? Quasi-itinerancy is the key property of the electrons in the weak Mott regime where the Mott gap is not large enough to fully localize the electron to one single lattice site and the electron can still be delocalized to a finite extent spatially due to the small charge gap. Electron quasi-itinerancy is believed to be the driving force for the possible spin liquid phase in the weak Mott regime for $\kappa-(\mathrm{ET})_{2} \mathrm{Cu}_{2}(\mathrm{CN})_{3}$ and $\mathrm{EtMe}_{3} \mathrm{Sb}\left[\mathrm{Pd}(\mathrm{dmit})_{2}\right]_{2}$ [11]. Over there, the electron quasi-itinerancy was found to generate the frustrated ring exchange interactions that suppress the magnetic orders. A similar kind of electron quasi-itinerancy [12-14] that emphasizes different outcomes of the charge fluctuations has been discussed in various spinels and osmate pyrochlores. Thus, besides the prevailing strong-coupling perspectives, the weak- to intermediate-coupling perspective is found to be both complementary and exciting. Reference [15] applied a slave-rotor mean-field theory to study the Mott transition in a series of rare-earth-based pyrochlore iridates, $R_{2} \mathrm{Ir}_{2} \mathrm{O}_{7}$. They discovered a topological band insulator in the 
noninteracting limit and a novel topological Mott insulator in the intermediate-coupling regime. Several other groups reexamined the problem with a more realistic Hamiltonian and discovered various magnetic ordered phases and an interesting Weyl semimetal phase that is located in the narrow regime separating the topological band insulator or metal phase from the strong-coupling Mott insulating phase [16-18]. Aligned with the above theoretical efforts, the experiments discovered that a metal-insulator transition in $R_{2} \mathrm{Ir}_{2} \mathrm{O}_{7}(R=\mathrm{Nd}, \mathrm{Sm}$, $\mathrm{Y}$, and $\mathrm{Eu}$ ) involves a magnetic ordering produced by the $5 d$ electrons in Ir [19-42]. Moreover, an exotic spin liquid metallic phase was also proposed experimentally for $\operatorname{Pr}_{2} \operatorname{Ir}_{2} \mathrm{O}_{7}$ $[43,44]$. Now, the Ir electrons are proposed as forming a Luttinger semimetal [45-47], while the Pr spin is proposed to be proximate to a transition between a $\mathrm{U}(1)$ spin liquid and an ordered spin ice [48-51].

Based on the existing theoretical [15-17,46-48,52-58] and experimental works, the true magnetic state of these Ir-based pyrochlore systems remains open. In this paper, we address this problem and provide some understanding. We primarily focus on the magnetic properties and avoid touching the band structure topology that has been invoked in early works. We first explore the magnetic properties of the Ir-based pyrochlore lattice in the strong-coupling regime. Physically, since the $5 d$ electron orbitals of $\mathrm{Ir}^{4+}$ are spatially extended, which enhances the electron bandwidth, these Ir-based systems are usually considered to be in the intermediate-coupling regime. Nevertheless, the SOC could enhance the correlation by suppressing the bandwidth [15]. Moreover, certain magnetic properties in the strong-coupling limit could persist to the intermediate-coupling regime even if the system is located in the intermediate-coupling regime. In the strongcoupling limit, the effective moments $J=1 / 2$ of the $\operatorname{Ir}^{4+}$ ions are coupled by the superexchange interaction. We analyze the symmetry-allowed exchange Hamiltonian, which includes three types of pairwise terms: Heisenberg exchange, antisymmetric Dzyaloshinskii-Moriya (DM) interaction, and symmetric pseudodipolar (PD) interaction. This model is equivalent to the one that was used for the interacting Kramers doublet for the rare-earth pyrochlores. In the mean-field phase diagram, we find five different ordered phases (see Sec. II): a "four-in-four-out" state, a continuously degenerate state spanned by two basis vectors $\left(\mathbf{v}_{1}, \mathbf{v}_{2}\right)$, a weakly ferromagnetic state (FM), and two coplanar states with spin oriented along the particular [110] directions. Almost all these ordered states have the magnetic wave vector $\mathbf{q}=0$. For the realistic exchange model obtained from an extended Hubbard model relevant for $R_{2} \mathrm{Ir}_{2} \mathrm{O}_{7}$, there are only two ordered phases, which are the four-in-four-out state and the continuously degenerate manifold spanned by two basis vectors $\left(\mathbf{v}_{1}, \mathbf{v}_{2}\right)$. For the latter, we find that the quantum fluctuation selects a noncoplanar spin configuration by a linear spin-wave expansion. This is the mechanism of quantum order by disorder. For the intermediate-coupling regime, we apply the self-consistent mean-field theory to study the microscopic Hubbard model and assume a general magnetic configuration except having the same magnetic cell as the crystallographic cell (or $\mathbf{q}=0$ ) order. Again, we find that the system is "fluctuating" within the continuously degenerate manifold spanned by $\left(\mathbf{v}_{1}, \mathbf{v}_{2}\right)$, and the electron kinetic energy selects the magnetic orders.
The electron kinetic energy is important here due to the quasi-itinerancy in the weak Mott regime. It is found that the magnetic orders in the strong-coupling regime persist into the intermediate-coupling regime. Since it is unclear which regime the actual system is proximate to, it is reasonable to think that the electron quasi-itinerancy is intertwined with the quantum order by disorder here.

In the following, we outline the main content of this paper. In Sec. II, we study a generic symmetry-allowed exchange Hamiltonian on the pyrochlore lattice with the effective spin$1 / 2$ originating from Kramers degeneracy, which is relevant for $R_{2} \mathrm{Ir}_{2} \mathrm{O}_{7}$ in the strong-coupling regime. In the exchange Hamiltonian, there are four symmetry-allowed coupling parameters: Heisenberg exchange $J_{0}, \mathrm{DM}$ interaction $D$, and $\Gamma_{1}, \Gamma_{2}$ for PD interaction. We analyze this Hamiltonian with the mean-field method in different parameter regimes. In many parts of the phase diagram, the ground state can be understood as simultaneously optimizing different terms of the Hamiltonian. In Sec. III, we derive a realistic exchange from the extended Hubbard model. Two limits with the dominant direct or indirect electron tunneling via intermediate oxygens are considered. In these two cases, we find that there is only one mean-field phase, which is the continuously degenerate manifold $\left(\mathbf{v}_{1}, \mathbf{v}_{2}\right)$. We then implement the linear spin-wave theory, and a noncoplanar ground state is favored by this quantum order-by-disorder mechanism. For the certain intermediate regime with comparable direct and indirect electron tunnelings, the four-in-four-out state is favored. We further explore the magnetic properties of the Hubbard model in the intermediate-coupling regime. By assuming a $\mathbf{q}=0$ magnetic structure, we implement a Hartree-Fock type of selfconsistent mean-field theory for the interaction. Finally, in Sec. IV, we discuss the relevant experiments and other related works.

\section{THE GENERIC EXCHANGE HAMILTONIAN}

In this section, we analyze the Ir-based pyrochlore lattice in the strong-coupling regime. In the strong-coupling limit, the local effective spin moments are coupled by an exchange Hamiltonian. For the effective spin-1/2 moment describing the local Kramers doublets, the exchange interaction is guaranteed to be pairwise. The generic exchange Hamiltonian has the following form:

$$
\mathcal{H}_{\mathrm{ex}}=\sum_{\langle i j\rangle} J_{0}\left(\mathbf{J}_{i} \cdot \mathbf{J}_{j}\right)+\mathbf{D}_{i j} \cdot\left(\mathbf{J}_{i} \times \mathbf{J}_{j}\right)+\Gamma_{i j}^{\mu \nu} J_{i}^{\mu} J_{j}^{\nu},
$$

where the nearest-neighbor interaction is assumed, $J_{0}$ is the isotropic Heisenberg exchange, $\mathbf{D}_{i j}$ describes the antisymmetric DM interaction, and $\Gamma_{i j}^{\mu \nu}$ is the symmetric PD interaction. This form of decomposition is well known in the much older literature of magnetism [59] but is not quite as popular among newer studies. Kitaev or any other anisotropic exchange interactions can be well cast into this form, as long as they are pairwise interactions. As a general rule of thumb, for systems with a weak SOC, the DM interaction is weaker than the Heisenberg part, and PD interaction is even weaker than DM interaction. For systems with strong SOC such as the iridates here, there is no general rule of thumb, and all the interactions could be of similar magnitudes. Thus, for most 


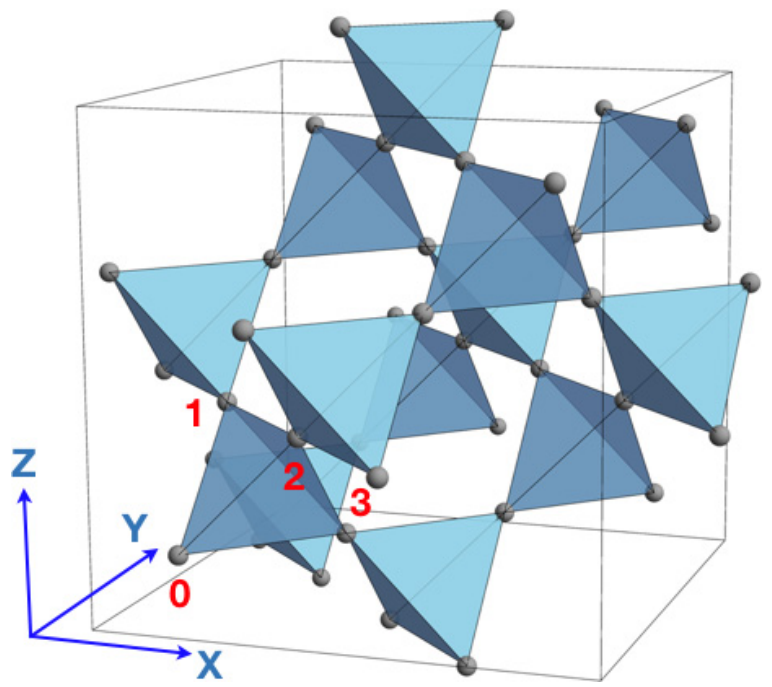

FIG. 1. The pyrochlore lattice in the global cubic coordinate system. " 0, ," "1," "2," and "3" label the four sublattices.

magnetic systems composed of $3 d$ transition-metal ions, the DM interaction and PD interaction are expected to be much weaker than the Heisenberg exchange and hence can be neglected at lowest-order approximation. For Ir-based magnets or other magnetic systems formed by $4 d / 5 d$ transition-metal ions, SOC is quite strong, and local moment is a mixture of spin and orbitals. As a result, the exchange interaction is usually very non-Heisenberg-like, and the anisotropic exchanges (such as DM and PD interactions) can be quite significant.

Throughout this section, we assume an antiferromagnetic Heisenberg part with $J_{0}>0$. Since most $R_{2} \mathrm{Ir}_{2} \mathrm{O}_{7}$ (and also spinel $A B_{2} X_{4}$ ) compounds have a space group $\mathrm{Fd} \overline{3} m$, this space-group symmetry further restricts the allowed forms of the DM interaction and PD interaction. Therefore, for the bond connecting sublattice 0 with sublattice 1 (see Fig. 1), we have

$$
\begin{gathered}
\mathbf{D}_{01}=D\left(0, \frac{1}{\sqrt{2}},-\frac{1}{\sqrt{2}}\right), \\
\Gamma_{01}=\left[\begin{array}{ccc}
-2 \Gamma_{1} & 0 & 0 \\
0 & \Gamma_{1} & -\Gamma_{2} \\
0 & -\Gamma_{2} & \Gamma_{1}
\end{array}\right],
\end{gathered}
$$

where the matrix $\Gamma_{01}$ is demanded to be symmetric and traceless, as the part of the full interaction that has a trace is taken care of by the Heisenberg interaction and the antisymmetric part is from the DM interaction. Exchange interactions on other bonds can be simply generated by cubic permutations.

Although the exchange Hamiltonian in Eq. (1) is introduced for an Ir-based pyrochlore lattice, it is widely applicable to other pyrochlore systems with the same symmetry properties as long as the local moment is a Kramers spin- $1 / 2$ doublet. Our results would also apply to these contexts as well. In fact, this model is equivalent to the one that was used for the rare-earth pyrochlore material $\mathrm{Yb}_{2} \mathrm{Ti}_{2} \mathrm{O}_{7}$, for which some detailed analyses are given in Refs. [60,61]. In those studies, a local coordinate system was used for each pyrochlore sublattice, and the local moment is the Kramers doublet of the $\mathrm{Yb}^{3+}$ ion, while here we are using a global cubic coordinate for the $\mathrm{Ir}^{4+}$ effective spin-1/2 moments. In Sec. II A, we analyze the mean-field ground states of this general Hamiltonian and understand the role of different anisotropic interactions.

\section{A. Role of Dzyaloshinskii-Moriya interaction}

Here, we consider the role of Dzyaloshinskii-Moriya interaction on top of the Heisenberg interaction and set $\Gamma_{1}=\Gamma_{2}=0$. Classically, it is well known that the pyrochlore lattice is the most frustrated lattice by having a macroscopic number of ground-state degeneracies with the nearest-neighbor Heisenberg model. The presence of the anisotropic exchange surely lifts the classical ground-state degeneracy. Reference [62] has already studied the role of DM interaction using mean-field theory and classical Monte Carlo simulation. Our mean-field analysis below, by treating the effective spin $\boldsymbol{J}_{i}$ as a classical vector, is consistent with their results. With a direct DM interaction that corresponds to $D<0$ in the present work, the ground state is twofold degenerate (related by time reversal) with the magnetic ordering wave vector $\mathbf{q}=\mathbf{0}$. The magnetic unit cell coincides with the crystallographic one, and the four spins on the unit cell are

$$
\begin{aligned}
\Psi & \equiv\left(\mathbf{J}_{0}, \mathbf{J}_{1}, \mathbf{J}_{2}, \mathbf{J}_{3}\right) \\
& =\frac{1}{\sqrt{3}}(111,1 \overline{1} \overline{1}, \overline{1} 1 \overline{1}, \overline{1} \overline{1} 1) .
\end{aligned}
$$

Here, we define a vector $\Psi$ for the four spin vectors on the elementary tetrahedron. This is the simple four-in-four-out state (see Fig. 2).

For the indirect DM interaction with $D>0$, DM interaction only partially lifts the ground-state degeneracy. There are two sets of ground states, coplanar and noncoplanar states, both of which have a magnetic wave vector $\mathbf{q}=\mathbf{0}$. The fourspin vector $\Psi$ of the coplanar ground states can be constructed as linear superpositions of the following two basis vectors $\mathbf{u}_{1}$ and $\mathbf{u}_{2}$ (or their equivalents under discrete symmetry operations):

$$
\begin{aligned}
& \mathbf{u}_{1}=(100,010,0 \overline{1} 0, \overline{1} 00), \\
& \mathbf{u}_{2}=(010, \overline{1} 00,100,0 \overline{1} 0) .
\end{aligned}
$$

The noncoplanar states are constructed from the following two basis vectors $\mathbf{v}_{1}$ and $\mathbf{v}_{2}$ (or their symmetry equivalents):

$$
\begin{aligned}
& \mathbf{v}_{1}=\frac{1}{\sqrt{2}}(\overline{1} 10, \overline{1} \overline{1} 0,110,1 \overline{1} 0), \\
& \mathbf{v}_{2}=\frac{1}{\sqrt{6}}(\overline{1} \overline{1} 2, \overline{1} 1 \overline{2}, 1 \overline{1} \overline{2}, 112) .
\end{aligned}
$$

Here, when only the first basis vector $\mathbf{v}_{1}$ is chosen, the ground state is a special coplanar state with spin oriented along different [110] lattice directions. Both the coplanar and noncoplanar degenerate ground-state manifolds have an accidental U(1) degeneracy with one continuous degree of freedom. This degenerate spin manifold is actually identical to the one that was proposed for the rare-earth pyrochlore $\mathrm{Er}_{2} \mathrm{Ti}_{2} \mathrm{O}_{7}$ and is selected via the quantum order-by-disorder mechanism [63]. 


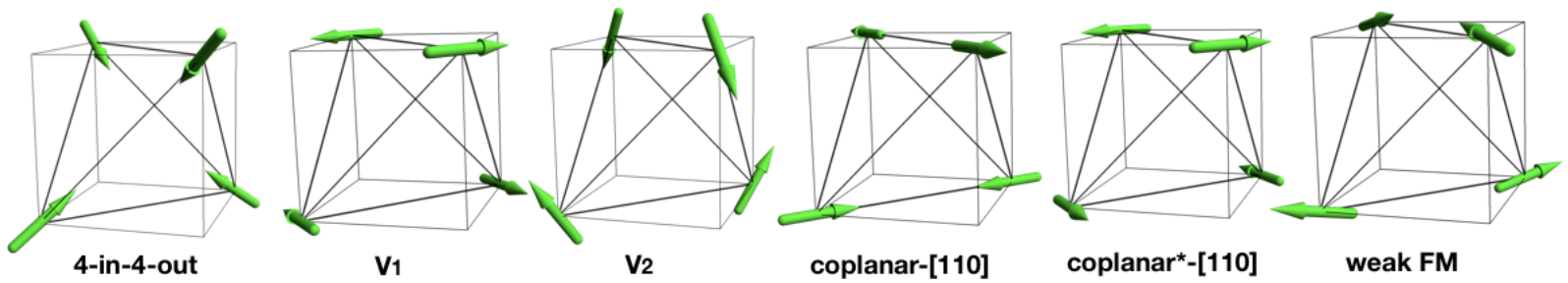

FIG. 2. The spin configuration on each sublattice for different phases.

\section{B. Role of pseudodipolar interaction: Case 1}

Here and in Sec. IIC, we study the role of the PD interaction. We first consider the regime with $D=0, \Gamma_{1} \neq 0, \Gamma_{2}=0$. For $\Gamma_{1}>0$, we find that optimal spin configurations have magnetic wave vector $\mathbf{q}=\mathbf{0}$. Even though the Hamiltonian breaks the spin rotation symmetry completely, the ground-state manifold has an accidental $\mathrm{O}(3)$ degeneracy. The four-spin vector $\Psi$ of the ground states is an arbitrary linear superposition of the following three basis vectors $\mathbf{w}_{1}, \mathbf{w}_{2}$, and $\mathbf{w}_{3}$ :

$$
\begin{aligned}
& \mathbf{w}_{1}=(100,100, \overline{1} 00, \overline{1} 00), \\
& \mathbf{w}_{2}=(010,0 \overline{1} 0,010,0 \overline{1} 0), \\
& \mathbf{w}_{3}=(001,00 \overline{1}, 00 \overline{1}, 001) .
\end{aligned}
$$

For $\Gamma_{1}<0$, to simultaneously optimize the energy and satisfy the hard spin constraint, there only exist two sets of ground states. One has the magnetic wave vector $\mathbf{q}=\mathbf{0}$. Similar to the case with $\Gamma_{1}>0$, the ground-state spin configuration has $\mathrm{O}(3)$ degeneracy, and the four-spin vector $\Psi$ is constructed from the following three basis vectors $\mathbf{z}_{1}, \mathbf{z}_{2}$, and $\mathbf{z}_{3}$ (or their symmetry equivalents):

$$
\begin{aligned}
& \mathbf{z}_{1}=(100, \overline{1} 00, \overline{1} 00,100) \\
& \mathbf{z}_{2}=(010,0 \overline{1} 0,0 \overline{1} 0,010), \\
& \mathbf{z}_{3}=(001,001,00 \overline{1}, 00 \overline{1}) .
\end{aligned}
$$

The other set of ground states has the magnetic wave vector $\mathbf{q}=2 \pi(100)$ or its cubic equivalents. Although the magnetic unit cell doubles the size of the crystallographic cell, the spin configuration can still be fully described within one tetrahedron, the four-spin vector $\Psi$ is given as

$$
\Psi=(\overline{1} 00,100, \overline{1} 00,100),
$$

and the spin configuration of other sites is generated from this and the ordering wave vector.

\section{Role of pseudodipolar interaction: Case 2}

Here, we consider the parameter regime with $D=0, \Gamma_{1}=0, \Gamma_{2} \neq 0$. For $\Gamma_{2}<0$, the ground state is the same as the case for $D<0$, which is the four-in-four-out state. For $\Gamma_{2}>0$, the anisotropy does not lift the classical degeneracy of the nearest-neighbor Heisenberg model on the pyrochlore lattice.

\section{With both Dzyaloshinskii-Moriya and pseudodipolar interactions}

In this section, we study the classical phase diagram when both of the two anisotropic exchanges are present. We start from the $D-\Gamma_{1}$ plane with $\Gamma_{2}=0$. The phase diagram is depicted in Fig. 3. In all the parts of the phase diagram, the magnetic wave vector is $\mathbf{q}=\mathbf{0}$. Most parts of the phase diagram can be understood as the intersection of two different ground-state manifolds separately favored by $D$ and $\Gamma_{1}$, which have already been discussed in detail in the previous sections.

For $D<0, \Gamma_{1}>0$, the four-in-four-out state is favored. For $D>0, \Gamma_{1}>0$, we have the classical ground states constructed as the linear superpositions of the same two basis vectors $\mathbf{v}_{1}$ and $\mathbf{v}_{2}$ that are introduced in Eqs. (7) and (8) for the case of $D>0$. For $D>0, \Gamma_{1}<0$, the ground state is a coplanar state with the spins pointing along different [110] directions (denoted as "coplanar-[110]" in Fig. 3), whose fourspin vectors $\Psi$ can be constructed from the basis vectors $\mathbf{u}_{1}$ and $\mathbf{u}_{2}$ in Eqs. (5) and (6),

$$
\Psi=\frac{1}{\sqrt{2}}(110, \overline{1} 10,1 \overline{1} 0, \overline{1} \overline{1} 0) .
$$

For $D<0, \Gamma_{1}<0$, the $D$-demanded and $\Gamma_{1}$-demanded ground-state manifolds have no overlap. We find that when $D<3 \sqrt{2} \Gamma_{1}$, DM interaction has more weight in the Hamiltonian and the ground state is the four-in-four-out state and, in the opposite case, the ground state is a coplanar state (denoted as "coplanar*-[110]" in Fig. 3) whose four-spin vector is given as

$$
\Psi=\frac{1}{\sqrt{2}}(1 \overline{1} 0, \overline{1} \overline{1} 0,110, \overline{1} 10) .
$$

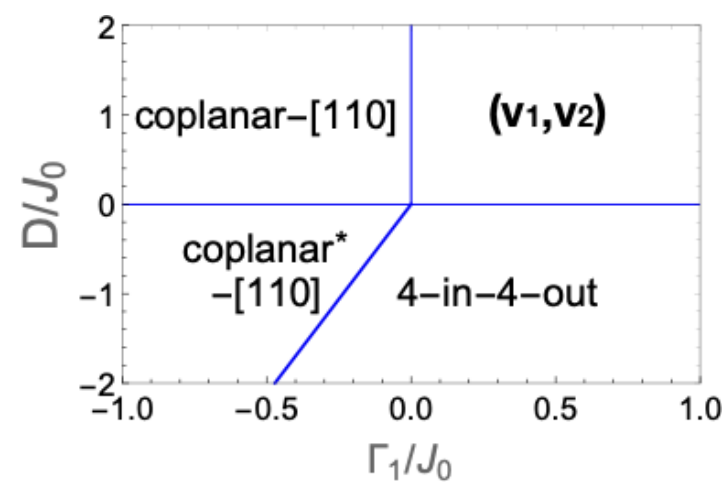

FIG. 3. The mean-field phase diagram in the $D-\Gamma_{1}$ plane with $\Gamma_{2}=0$. The corresponding spin configurations are found in Fig. 2 . 


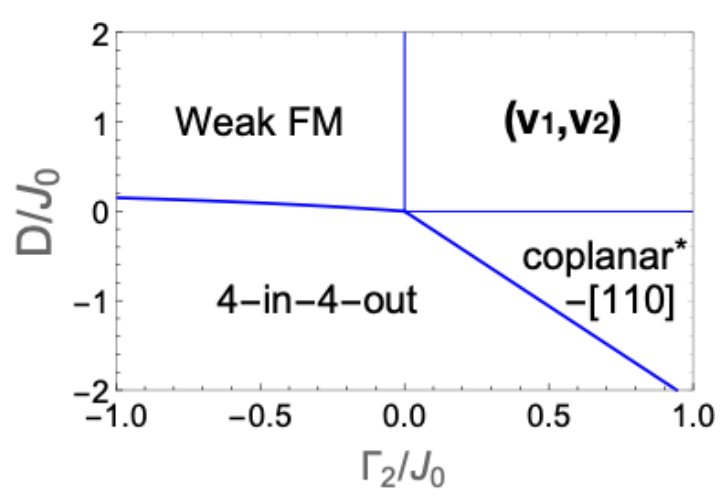

FIG. 4. The mean-field phase diagram in the $D-\Gamma_{2}$ plane with $\Gamma_{1}=0$. The corresponding spin configurations are found in Fig. 2.

Note that this coplanar state is distinct from the coplanar-[110] state found for $D>0, \Gamma_{1}<0$.

Now we discuss the ground states in the $D-\Gamma_{2}$ plane with $\Gamma_{1}=0$. The phase diagram is depicted in Fig. 4. The magnetic wave vector is $\mathbf{q}=0$ everywhere in the phase diagram.

For $D<0, \Gamma_{2}<0$, the ground state is simply the fourin-four-out state. For $D>0, \Gamma_{2}>0$, the ground state is an arbitrary linear superposition of the basis vectors $\mathbf{v}_{1}$ and $\mathbf{v}_{2}$ in Eqs. (7) and (8). In the regime of $D>0, \Gamma_{2}<0$, there exist two phases. When $D>D_{c 1}\left(\Gamma_{2}\right)$ with

$$
D_{c 1}\left(\Gamma_{2}\right)=\frac{\sqrt{2}}{6}\left(3 J_{0}-2 \Gamma_{2}-\sqrt{9 J_{0}^{2}-6 J_{0} \Gamma_{2}+4 \Gamma_{2}^{2}}\right),
$$

the ground state turns out to be weakly ferromagnetic and is denoted as "weak FM" in Fig. 4. The four-spin vectors of the magnetic unit cell are parametrized as

$$
\Psi=\cos \theta y_{1}+\sin \theta y_{2}
$$

with

$$
\begin{gathered}
y_{1}=\frac{1}{\sqrt{2}}(\overline{1} \overline{1} 0,1 \overline{1} 0, \overline{1} 10,110), \\
y_{2}=(001,001,001,001),
\end{gathered}
$$

and the angular variable $\theta$ satisfies

$$
\begin{aligned}
& \cos 2 \theta=\frac{4 J_{0}+\sqrt{2} D-\Gamma_{2}}{\sqrt{\left(4 J_{0}+\sqrt{2} D-\Gamma_{2}\right)^{2}+8 \Gamma_{2}^{2}}}, \\
& \sin 2 \theta=\frac{-2 \sqrt{2} \Gamma_{2}}{\sqrt{\left(4 J_{0}+\sqrt{2} D-\Gamma_{2}\right)^{2}+8 \Gamma_{2}^{2}}} .
\end{aligned}
$$

When $D<D_{c 1}\left(\Gamma_{2}\right)$, the ground state is the four-in-four-out state.

In the region of $D<0, \Gamma_{2}>0$, there also exist two phases. When $D<D_{c 2}\left(\Gamma_{2}\right)$ with $D_{c 2}\left(\Gamma_{2}\right)$ given by

$$
D_{c 2}=-\frac{3 \sqrt{2} \Gamma_{2}}{2}
$$

and the DM interaction is dominant and negative, the ground state is the four-in-four-out state. When $D>D_{c 2}\left(\Gamma_{2}\right)$, a coplanar state with spins pointing along various [110] directions is favored, and the four-spin vector $\Psi$ is the same as the
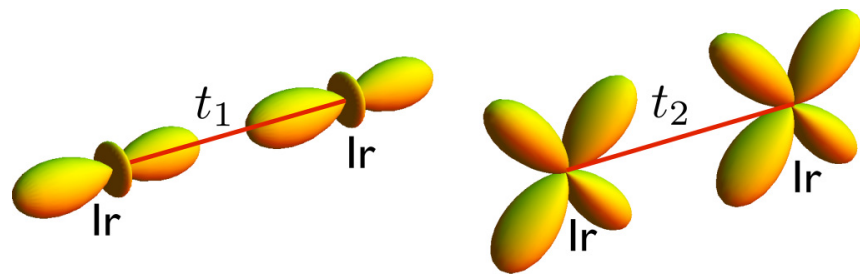

FIG. 5. The direct electron tunneling between the Ir atoms. Left: the $\sigma$ bonding with tunneling amplitude $t_{1}$. Right: the $\pi$ bonding with tunneling amplitude $t_{2}$.

one introduced in Eq. (17) and its symmetry equivalent. Hence we also denote this coplanar state as coplanar*-[110] in Fig. 4.

\section{MAGNETISM FROM ELECTRON QUASI-ITINERANCY}

Having understood the role of each anisotropic exchange for the generic exchange Hamiltonian in Sec. II, in this section we discuss the physical exchange Hamiltonian derived perturbatively from the microscopic parent Hubbard model and from there approach the magnetic states in the intermediatecoupling regime. We analyze the possible magnetic ground states for the compound $R_{2} \mathrm{Ir}_{2} \mathrm{O}_{7}$.

\section{A. Hubbard model and exchange}

We assume that the on-site SOC is strong enough so that the lower $J=3 / 2$ bands are completely filled and the upper $J=1 / 2$ bands are half filled. This approximation misses the hybridization between the $J=1 / 2$ and $J=3 / 2$ bands, and this process may lead to some interesting properties and needs to be addressed in future. The electrons can tunnel from one $\mathrm{Ir}^{4+}$ ion to neighboring $\mathrm{Ir}^{4+}$ ions either directly or indirectly via the $p$ orbitals of the intermediate oxygen ions $[15,17]$. Since $5 d$ electron orbitals are spatially extended, the direct tunneling of electrons might be equally as important as the indirect tunneling. With electrons locally projected onto the $J=1 / 2$ basis, one can write down a minimal Hubbard model [17]

$$
\mathcal{H}=\sum_{\langle i j\rangle}\left[\left(\mathcal{T}_{i j, \alpha \beta}^{d}+\mathcal{T}_{i j, \alpha \beta}^{i d}\right) d_{i \alpha}^{\dagger} d_{j \beta}+\text { H.c. }\right]+\sum_{i} U n_{i, \uparrow} n_{i, \downarrow},
$$

in which only the nearest-neighbor tunneling term is included, $d_{i \alpha}^{\dagger}\left(d_{i \alpha}\right)$ is the creation (annihilation) operator for an electron on effective spin state $\left|J=1 / 2, J^{z}=\alpha\right\rangle$ at site $i$, and $n_{i \sigma} \equiv d_{i \sigma}^{\dagger} d_{i \sigma}$ measures the electron number with spin $\sigma$ at site $i$. In Eq. (25), $\mathcal{T}^{d}$ and $\mathcal{T}^{i d}$ are the tunneling matrices for the direct and indirect processes, respectively.

For the direct tunneling processes, there exist two types of tunneling amplitudes: the $\sigma$-bonding type, $t_{1}$, and the $\pi$ bonding type, $t_{2}$ (see Fig. 5) [17]. Moreover, it is expected from the orbital overlaps that $t_{2}$ has a different sign from $t_{1}$. In the limit of dominant direct tunneling, standard secondorder perturbation yields the exchange couplings introduced in Eq. (1),

$$
J_{0}=\frac{603 t_{1}^{2}-58296 t_{1} t_{2}+248368 t_{2}^{2}}{2834352 U},
$$




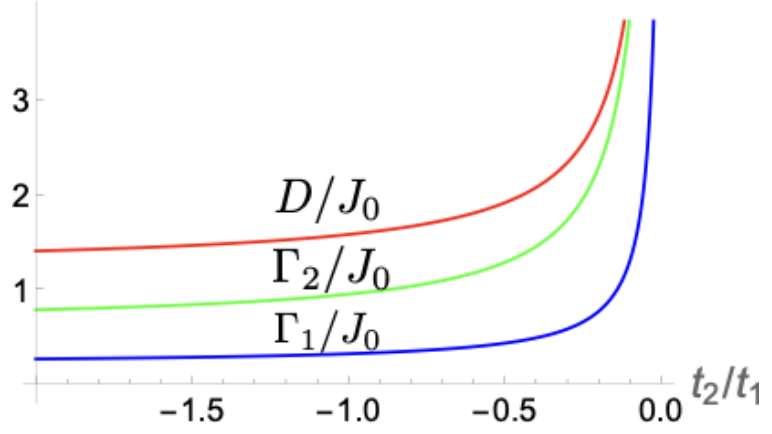

FIG. 6. The dependence of anisotropic couplings on the ratio between the $\pi$-bonding amplitude $t_{2}$ and the $\sigma$-bonding amplitude $t_{1}$. In the plot, from top to bottom, the curves are $D / J_{0}, \Gamma_{1} / J_{0}$, and $\Gamma_{2} / J_{0}$.

$$
\begin{gathered}
D=\frac{5 \sqrt{2}\left(153 t_{1}^{2}-1356 t_{1} t_{2}+2528 t_{2}^{2}\right)}{118098 U}, \\
\Gamma_{1}=\frac{50\left(9 t_{1}^{2}-48 t_{1} t_{2}+64 t_{2}^{2}\right)}{177147 U}, \\
\Gamma_{2}=3 \Gamma_{1} .
\end{gathered}
$$

It turns out that the DM interaction has the most weight in the exchange Hamiltonian. As $J_{0}$ is assured to be positive in Eq. (29), we depict the ratios $D / J_{0}, \Gamma_{1} / J_{0}$, and $\Gamma_{2} / J_{0}$ in Fig. 6 .

In contrast, the indirect tunneling process is described by one single tunneling amplitude $t$. [15] When it is dominant, the exchange couplings are given by

$$
\begin{gathered}
J_{0}=\frac{49132 t^{2}}{177147 U}, \\
D=\frac{7280 \sqrt{2} t^{2}}{59049 U}, \\
\Gamma_{1}=\frac{1568 t^{2}}{177147 U}, \\
\Gamma_{2}=3 \Gamma_{1} .
\end{gathered}
$$

It is important to note that although we find $\Gamma_{2}=3 \Gamma_{1}$ for both limits studied above, this relation is not protected by symmetry and will break down if a more realistic model is assumed. Although we find that $J_{0}, D, \Gamma_{1}$, and $\Gamma_{2}$ are all positive for the two limits studied above, this result actually breaks down when both direct and indirect tunnelings are included. As plotted in Fig. 7 for the case of $t_{2}=-t_{2} / 3$, the Heisenberg exchange $J_{0}$ and DM interaction $D$ both change sign for certain intermediate ranges of $t_{1} / t$. This indicates that different magnetic order may emerge in the intermediate regimes of $t_{1} / t$.

\section{B. Ground states of the exchange Hamiltonian}

In Sec. III A, we have explicitly derived the exchange Hamiltonian from the Hubbard model. For both exchanges in the limit of the dominant direct or indirect tunneling, the coupling parameters $J_{0}, D, \Gamma_{1}$, and $\Gamma_{2}$ are found to be positive. For this parameter regime, we are ready to show using the mean-field theory and/or directly observe from the phase

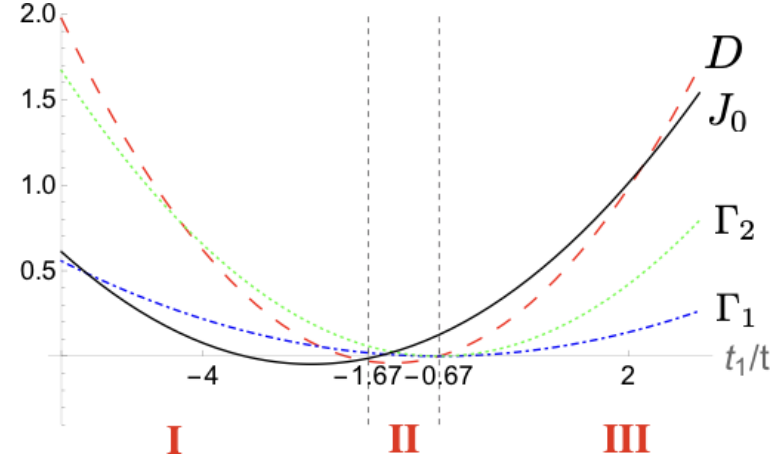

FIG. 7. The dependence of couplings on the ratio between the direct tunneling and the indirect tunneling. In this figure, we have set $t_{2}=-2 t_{1} / 3$. The ground state of region II is the four-in-four-out state. For regions I and III, the ground state is degenerate and spanned by the basis vectors $\mathbf{v}_{1}$ and $\mathbf{v}_{2}$. The two dashed vertical lines are the phase boundaries separating the four-in-four-out state in region II from the $\left(\mathbf{v}_{1}, \mathbf{v}_{2}\right)$ manifold in regions I and III. The units of the vertical axis are set to be $t^{2} / U$.

diagrams depicted in Figs. 3 and 4 that the mean-field classical ground-state manifold is continuously degenerate and is spanned by the two basis vectors $\mathbf{v}_{1}$ and $\mathbf{v}_{2}$ [see Eqs. (7) and (8)]. As shown in Fig. 7, there is a region in which the DM interaction $D$ changes sign that may favor the four-in-four-out state as the classical ground state in that region. After a complete calculation, we find the phase diagram that is depicted in Fig. 7. Region II develops the four-in-four-out ground state. Regions I and III have the degenerate ground-state manifold $\left(\mathbf{v}_{1}, \mathbf{v}_{2}\right)$. Remarkably, the phase boundaries between region II and regions I and III are exactly the same as those obtained from a self-consistent mean-field calculation for the intermediate-coupling regime in the calculation below and the one in Ref. [17].

This continuous degeneracy of the $\left(\mathbf{v}_{1}, \mathbf{v}_{2}\right)$ ground-state manifold will be lifted if the quantum fluctuation is included. We study this quantum order-by-disorder effect using the linear spin-wave theory. We express the classical four-spin vectors as

$$
\Psi=\cos \phi \mathbf{v}_{1}+\sin \phi \mathbf{v}_{2},
$$

where $\phi$ parametrizes the orientation of the spin vectors. Then we introduce the Holstein-Primakoff bosons,

$$
\begin{gathered}
\mathbf{J}_{i} \cdot \hat{m}_{i}=J-a_{i}^{\dagger} a_{i}, \\
\mathbf{J}_{i} \cdot \hat{n}_{i}=\frac{\sqrt{2 J}}{2}\left(a_{i}+a_{i}^{\dagger}\right), \\
\mathbf{J}_{i} \cdot\left(\hat{m}_{i} \times \hat{n}_{i}\right)=\frac{\sqrt{2 J}}{2 i}\left(a_{i}-a_{i}^{\dagger}\right),
\end{gathered}
$$

where $\hat{m}_{i}$ is the unit vector describing the spin orientation of classical spin order at site $i$ and $\hat{n}_{i}$ is a unit vector that is normal to $\hat{n}_{i}$ but within the plane spanned by $\mathbf{v}_{1}$ and $\mathbf{v}_{2}$. Plugging the above relations into the exchange Hamiltonian, one is ready to write the quadratic spin wave 


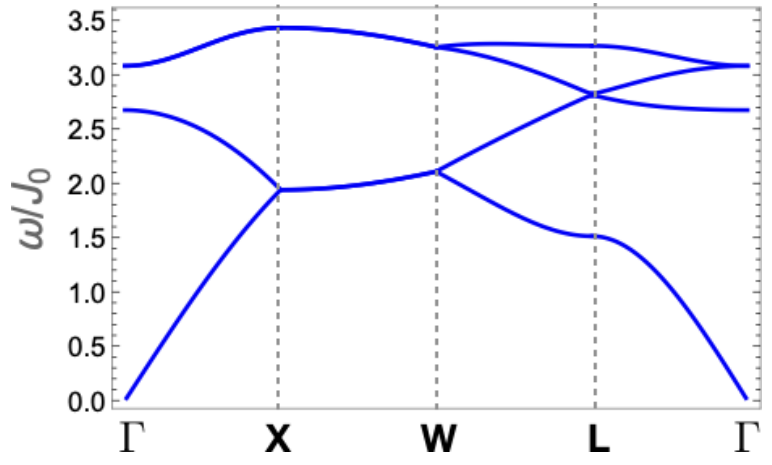

FIG. 8. The magnon dispersion along the high-symmetry momentum direction $\Gamma$-X-W-L- $\Gamma$. The parameters in this figure are set to be $D=0.5 J_{0}, \Gamma_{1}=0.2 J_{0}, \Gamma_{2}=0.3 J_{0}$. The gapless mode at the $\Gamma$ point is an artifact of the linear spin-wave theory.

Hamiltonian,

$$
\begin{aligned}
\mathcal{H}_{\mathrm{sw}}= & \sum_{\mathbf{k}}\left[A_{i j}(\mathbf{k}) a_{i}^{\dagger}(\mathbf{k}) a_{j}(\mathbf{k})+B_{i j}(\mathbf{k}) a_{i}(-\mathbf{k}) a_{j}(\mathbf{k})\right. \\
& \left.+B_{i j}^{*}(\mathbf{k}) a_{i}^{\dagger}(\mathbf{k}) a_{j}^{\dagger}(-\mathbf{k})\right]+E_{\mathrm{cl}},
\end{aligned}
$$

in which $E_{\mathrm{cl}}$ is the classical ground-state energy and $A_{i j}$ and $B_{i j}$ satisfy

$$
\begin{aligned}
& A_{i j}(\mathbf{k})=A_{i j}^{*}(\mathbf{k}), \\
& B_{i j}(\mathbf{k})=B_{j i}(-\mathbf{k})
\end{aligned}
$$

and are given in the Appendix. From the quadratic spin-wave Hamiltonian, we obtain the quantum zero-point energy, which is found to be optimized by the noncoplanar spin configuration $\mathbf{v}_{2}$ [see Eq. (8)] with $\phi=\pi / 2$ (and its symmetry equivalents). We also find that the magnon spectrum (see Fig. 8) is gapless at the $\Gamma$ point, which originates from the continuous degeneracy of the classical ground states. This gapless mode is not supposed to remain if the anharmonic effects beyond the linear spin-wave theory are included, as the gapless feature is not protected by any continuous symmetry of the Hamiltonian. A minigap would appear if a full calculation is performed.

\section{Hubbard model and electron quasi-itinerancy in the intermediate-coupling regime}

In the previous sections, we have analyzed the magnetic ground states of the Ir-based pyrochlore lattice for $R_{2} \mathrm{Ir}_{2} \mathrm{O}_{7}$ in the strong-coupling regime. We find that even though the classical mean-field ground states are continuously degenerate for the exchange derived from the Hubbard model, all the ground states have a magnetic wave vector $\mathbf{q}=\mathbf{0}$. It is known that the SOC twists the electron motion and reduces the electron bands. Although the large spatial extension of the $5 d$ electrons reduces the electron correlation, as the bandwidth is also reduced, it is then not quite obvious where the actual physical system is located. Thus it is legitimate for us to tackle the system from the strong correlation to the intermediate correlation by reducing the correlation strength. The knowledge that we have learned from the strong-coupling regime may be extended to the intermediate regime. Moreover, the existing experiments on $\mathrm{Eu}_{2} \mathrm{Ir}_{2} \mathrm{O}_{7}, \mathrm{Nd}_{2} \mathrm{Ir}_{2} \mathrm{O}_{7}, \mathrm{~Tb}_{2} \mathrm{Ir}_{2} \mathrm{O}_{7}$, and $\mathrm{Sm}_{2} \mathrm{Ir}_{2} \mathrm{O}_{7}$ suggest a $\mathbf{q}=\mathbf{0}$ magnetic order $[24,25,27,39]$. In this section, we study the magnetic properties of the Hubbard model in the intermediate-coupling regime by a self-consistent meanfield theory. Based on the results from the strong-correlation regime, we assume that the magnetic order in this regime also has a magnetic wave vector $\mathbf{q}=\mathbf{0}$. To implement the mean-field theory, we decouple the Hubbard $U$ interaction as

$$
\begin{aligned}
U n_{i, \uparrow} n_{i, \downarrow} & =-\frac{2 U}{3} \mathbf{J}_{i}^{2}+\frac{U}{6} n_{i} \\
& \rightarrow-\frac{2 U}{3}\left(2\left\langle\mathbf{J}_{i}\right\rangle \cdot \mathbf{J}_{i}-\left\langle\mathbf{J}_{i}\right\rangle^{2}\right)+\frac{U}{6} n_{i},
\end{aligned}
$$

in which $n_{i}$ is the electron number at site $i$ and $\mathbf{J}_{i}=\sum_{\alpha \beta} d_{i \alpha}^{\dagger} \sigma_{\alpha \beta} d_{i \beta} / 2$ is the operator for the effective spin moment. With this decoupling, the mean-field Hamiltonian is quadratic with

$$
\begin{aligned}
H_{\mathrm{MF}} \equiv & \sum_{\langle i j\rangle}\left[\left(\mathcal{T}_{i j, \alpha \beta}^{d}+\mathcal{T}_{i j, \alpha \beta}^{i d}\right) d_{i \alpha}^{\dagger} d_{j \beta}+\text { H.c. }\right] \\
& -\sum_{i} \frac{4 U}{3}\left\langle\mathbf{J}_{i}\right\rangle \cdot \mathbf{J}_{i}+\cdots
\end{aligned}
$$

where "..." refers to the unessential terms that do not involve the electron operators. We then diagonalize the mean-field Hamiltonian and solve for the magnetic order of each sublattice self-consistently. Our results for the magnetic orders can be found in Fig. 2. In region II, the calculation quickly converges to the four-in-four-out magnetic order. For regions I and III, the calculation does not quickly converge. After a few steps, the magnetic order from the self-consistent calculation actually drops into the continuous manifold that is spanned by the four-spin vectors $\mathbf{v}_{1}$ and $\mathbf{v}_{2}$ and then fluctuates within this manifold without seeing a quick convergence. To resolve the magnetic orders in these two regions, we perform a different calculation below that may be illuminating. The self-consistent calculation tells us that the magnetic orders can be parametrized as

$$
\begin{aligned}
\Psi(\phi) & =\left(\mathbf{J}_{0}, \mathbf{J}_{1}, \mathbf{J}_{2}, \mathbf{J}_{3}\right) \\
& =M\left(\cos \phi \mathbf{v}_{1}+\sin \phi \mathbf{v}_{2}\right),
\end{aligned}
$$

where the order parameter $M$ depends on the dimensionless parameter $U / t$ that measures the strength of the interaction. For a given $U / t$, the magnetic order parameter $M$ is fixed. The self-consistent calculation was unable to quickly converge the angular parameter $\phi$, which is the task to be fulfilled. We are ready to see that the task boils down to optimizing the kinetic energy in the mean-field Hamiltonian $H_{\mathrm{MF}}$, i.e.,

$$
\langle\Psi(\phi)| \sum_{\langle i j\rangle}\left[\left(\mathcal{T}_{i j, \alpha \beta}^{d}+\mathcal{T}_{i j, \alpha \beta}^{i d}\right) d_{i \alpha}^{\dagger} d_{j \beta}+\text { H.c. }\right]|\Psi(\phi)\rangle .
$$

The spirit of this calculation scheme is a bit similar to the double exchange. In the double exchange, the itinerant electron is coupled with the local moments with ferromagnetic Kondo or Hund coupling, and the magnetic order is established by optimizing the kinetic energy of the itinerant electrons and the exchange energy of the local moments [64]. In the doped manganites, to gain the kinetic energy, the local moments twist themselves from the spin configuration favored by the 
exchange energy. Another possibly electron-kinetic-energydriven magnetism was proposed for the doped van der Waals antiferromagnet $\mathrm{CeTe}_{3}$ [65] and was refereed as fermionic order by disorder. For our case here, the electron kinetic energy is optimized within the background of the magnetism that operates on the continuously degenerate manifold. Our calculation suggests the selection of $\pi / 2$ for the angle $\phi$ for all $U>0$. We find that the kinetic energy stabilizes the noncoplanar state with $\phi=\pi / 2$. Although this mechanism of breaking the continuous degeneracy by optimizing the kinetic energy is qualitatively different from the quantum order by disorder discussed in Sec. III B, the magnetic order from both mechanisms turns out to be identical, and the phase boundaries separating different ordered phases are also remarkably identical for both mechanisms. These results suggest that the magnetic orders in the intermediate- and the strong-coupling regimes may be continuously connected.

\section{DISCUSSION}

To summarize, we have studied the magnetic ground states for the Ir-based pyrochlore lattice in both intermediate- and strong-coupling regimes. Various classical ground states are identified for the generic exchange Hamiltonian in the strongcoupling limit. These results can be further applied to other magnetic systems on the pyrochlore lattice. We find that the magnetic orders in the intermediate- and strong-coupling regimes for the pyrochlore iridates turn out to be identical.

Experiments on the pyrochlore iridates have rapidly evolved [19-42]. There exists a large body of experimental work, and the review papers on this topic can provide more information to interested readers [1-4]. Instead of delving into a few specific experimental results and details, we here make some experimental suggestions based on the theoretical calculations in our work. In the strong-coupling analysis, there exists a broad parameter regime in which the magnetic order is realized from the quantum order-by-disorder mechanism. Once the particular magnetic order with the ordering wave vector $\mathbf{q}=0$ and the spins orientating along the vector $\mathbf{v}_{2}$ in Eq. (8) is realized, one can check whether the excitation spectrum and thermodynamic properties are consistent with the theoretical results. A qualitative feature in the magnetic excitation spectrum is the almost gapless mode at the $\Gamma$ point (see Fig. 8 and the explanation in Sec. III C). A consequence for the thermodynamics is the nearly $\sim T^{3}$ temperature dependence in the specific heat at temperatures above the minigap energy. In the intermediate-coupling scenario, the interaction and the charge gap are not very large compared with the bandwidth. Although the same magnetic order persists to the intermediate-coupling regime, the quantum order-bydisorder mechanism is expected to break down. If one uses the local-moment language and relies on the exchange interaction, one necessarily needs to invoke further neighbor exchanges and even the ring exchange interactions. These extra interactions modify the original pairwise nearest-neighbor exchange model and will break the original applicability of the quantum order by disorder here. A surprising result in our self-consistent calculation in Sec. IIIC is that the magnetic order quickly falls into the degenerate manifold spanned by $\mathbf{v}_{1}$ and $\mathbf{v}_{2}$, and then we use the electron kinetic energy to break the degeneracy and select the magnetic order. This indicates that the degenerate manifold could be readily accessible if the system is activated by a small energy. A pump-probe measurement of the magnetic properties of the system would be helpful in this regard.

Finally, the weak Mott regime with quasi-itinerant electrons might be relevant for many other $4 d / 5 d$ materials. The effect should be considered if the charge gap is not very large. It is very likely that many $4 d / 5 d$ magnets would be located in this regime. Even the square-lattice material $\mathrm{Sr}_{2} \mathrm{IrO}_{4}$ was believed to be proximate to a Mott transition [6]. The wellknown $\alpha-\mathrm{RuCl}_{3}$ has a relatively weak charge gap [66-69], even though the existing theoretical analysis mostly starts from a pairwise superexchange interaction between the effective spin- $1 / 2$ moments. The interlayer ring exchange, due to the weak Mott gap and the electron quasi-itinerancy, could be responsible for the anomalous thermal Hall effect in $\alpha-\mathrm{RuCl}_{3}$ for the magnetic field in the honeycomb plane and parallel to the zigzag ordering axis [70-72], where the interlayer magnetic flux could be experienced by the material.

\section{ACKNOWLEDGMENTS}

We thank Xu Ping Yao and Dr. Fei Ye Li for help with Figs. 1 and 2. We acknowledge the hospitality of Aspen Center for Physics during the ultracold atom program in the summer of 2011 when and where this work was carried out. We especially thank Michael Hermele for discussions around that time period. G.C. is supported by Ministry of Science and Technology of China Grants No. 2018YFGH000095, No. 2016YFA0301001, and No. 2016YFA0300500, by Shanghai Municipal Science and Technology Major Project Grant No. 2019SHZDZX04, and by Research Grants Council of Hong Kong General Research Fund Grant No. 17303819. The part of this work performed in Boulder was supported by DOE Award No. desc0003910. X.W. is supported by MOST Grant No. 2016YFA0300501 and NSFC Grant No. 11974244 and additionally by a Shanghai talent program.

\section{APPENDIX: THE LINEAR SPIN-WAVE THEORY}

In Sec. III B of the main text, the couplings $A_{i j}(\mathbf{k})$ and $B_{i j}(\mathbf{k})$ in the spin-wave Hamiltonian for the magnetic orders given by the basis vector $\mathbf{v}_{2}$ are listed as follows:

$$
\begin{aligned}
A_{00}(\mathbf{k}) & =A_{11}(\mathbf{k}) \\
& =A_{22}(\mathbf{k})=A_{33}(\mathbf{k})=c_{1}, \\
A_{12}(\mathbf{k}) & =\frac{1}{24}\left(1+e^{-i\left(k_{y}+k_{z}\right) / 2}\right) c_{2}, \\
A_{13}(\mathbf{k}) & =\frac{1}{24}\left(1+e^{-i\left(k_{x}+k_{z}\right) / 2}\right) c_{2}, \\
A_{14}(\mathbf{k}) & =\frac{1}{12}\left(1+e^{-i\left(k_{x}+k_{y}\right) / 2}\right) c_{3}, \\
A_{23}(\mathbf{k}) & =\frac{1}{12}\left(1+e^{-i\left(k_{x}-k_{y}\right) / 2}\right) c_{3}, \\
A_{24}(\mathbf{k}) & =\frac{1}{24}\left(1+e^{-i\left(k_{x}-k_{z}\right) / 2}\right) c_{2}, \\
A_{34}(\mathbf{k}) & =\frac{1}{24}\left(1+e^{-i\left(k_{y}-k_{z}\right) / 2}\right) c_{2}
\end{aligned}
$$


and

$$
\begin{aligned}
& B_{12}(\mathbf{k})=\frac{1}{24}\left(1+e^{-i\left(k_{y}+k_{z}\right) / 2}\right) c_{4}, \\
& B_{13}(\mathbf{k})=\frac{1}{24}\left(1+e^{-i\left(k_{x}+k_{z}\right) / 2}\right) c_{4}^{*}, \\
& B_{14}(\mathbf{k})=\frac{1}{12}\left(1+e^{-i\left(k_{x}+k_{y}\right) / 2}\right) c_{5}, \\
& B_{23}(\mathbf{k})=\frac{1}{12}\left(1+e^{-i\left(k_{x}-k_{y}\right) / 2}\right) c_{5}, \\
& B_{24}(\mathbf{k})=\frac{1}{24}\left(1+e^{-i\left(k_{x}-k_{z}\right) / 2}\right) c_{4}^{*}, \\
& B_{34}(\mathbf{k})=\frac{1}{24}\left(1+e^{-i\left(k_{y}-k_{z}\right) / 2}\right) c_{4},
\end{aligned}
$$

in which we have set $J=1 / 2$ and the coefficients are given as

$$
\begin{gathered}
c_{1}=J_{0}+\sqrt{2} D+4 \Gamma_{1}+\Gamma_{2}, \\
c_{2}=-2 J_{0}+\sqrt{2} D-17 \Gamma_{1}+4 \Gamma_{2}, \\
c_{3}=-4 J_{0}+2 \sqrt{2} D-7 \Gamma_{1}-\Gamma_{2}, \\
c_{4}=-(2+4 i \sqrt{6}) J_{0}+(7 \sqrt{2}-2 i \sqrt{3}) D \\
+(1+2 i \sqrt{6}) \Gamma_{1}+(4+2 i \sqrt{6}) \Gamma_{2}, \\
c_{5}=2 J_{0}+2 \sqrt{2} D-\Gamma_{1}+5 \Gamma_{2} .
\end{gathered}
$$

Other entries of $A_{i j}(\mathbf{k})$ and $B_{i j}(\mathbf{k})$ are either zero or obtained by the relations in Eq. (40).
[1] W. Witczak-Krempa, G. Chen, Y. B. Kim, and L. Balents, Correlated quantum phenomena in the strong spin-orbit regime, Annu. Rev. Condens. Matter Phys. 5, 57 (2014).

[2] J. G. Rau, E. K.-H. Lee, and H.-Y. Kee, Spin-orbit physics giving rise to novel phases in correlated systems: Iridates and related materials, Annu. Rev. Condens. Matter Phys. 7, 195 (2016).

[3] H. Takagi, T. Takayama, G. Jackeli, G. Khaliullin, and S. E. Nagler, Concept and realization of Kitaev quantum spin liquids, Nat. Rev. Phys. 1, 264 (2019).

[4] R. Schaffer, E. K.-H. Lee, B.-J. Yang, and Y. B. Kim, Recent progress on correlated electron systems with strong spin-orbit coupling, Rep. Prog. Phys. 79, 094504 (2016).

[5] Y. Okamoto, M. Nohara, H. Aruga-Katori, and H. Takagi, SpinLiquid State in the $S=1 / 2$ Hyperkagome Antiferromagnet $\mathrm{Na}_{4} \mathrm{Ir}_{3} \mathrm{O}_{8}$, Phys. Rev. Lett. 99, 137207 (2007).

[6] G. Cao, J. Bolivar, S. McCall, J. E. Crow, and R. P. Guertin, Weak ferromagnetism, metal-to-nonmetal transition, and negative differential resistivity in single-crystal $\mathrm{Sr}_{2} \mathrm{IrO}_{4}$, Phys. Rev. B 57, R11039 (1998).

[7] G. Chen and L. Balents, Spin-orbit effects in $\mathrm{Na}_{4} \mathrm{Ir}_{3} \mathrm{O}_{8}$ : A hyper-kagome lattice antiferromagnet, Phys. Rev. B 78, 094403 (2008).

[8] G. Jackeli and G. Khaliullin, Mott Insulators in the Strong SpinOrbit Coupling Limit: From Heisenberg to a Quantum Compass and Kitaev Models, Phys. Rev. Lett. 102, 017205 (2009).

[9] B. J. Kim, H. Ohsumi, T. Komesu, S. Sakai, T. Morita, H. Takagi, and T. Arima, Phase-sensitive observation of a spinorbital Mott state in $\mathrm{Sr}_{2} \mathrm{IrO}_{4}$, Science 323, 1329 (2009).

[10] J. Chaloupka, G. Jackeli, and G. Khaliullin, Kitaev-Heisenberg Model on a Honeycomb Lattice: Possible Exotic Phases in Iridium Oxides $A_{2} \mathrm{IrO}_{3}$, Phys. Rev. Lett. 105, 027204 (2010).

[11] S.-S. Lee and P. A. Lee, U(1) Gauge Theory of the Hubbard Model: Spin Liquid States and Possible Application to $\kappa$-(BEDT-TTF) ${ }_{2} \mathrm{Cu}_{2}(\mathrm{CN})_{3}$, Phys. Rev. Lett. 95, 036403 (2005).

[12] Y. Kato, G.-W. Chern, K. A. Al-Hassanieh, N. B. Perkins, and C. D. Batista, Orbital Disorder Induced by Charge Fluctuations in Vanadium Spinels, Phys. Rev. Lett. 108, 247215 (2012).

[13] G.-W. Chern and C. D. Batista, Spin Superstructure and Noncoplanar Ordering in Metallic Pyrochlore Magnets with Degenerate Orbitals, Phys. Rev. Lett. 107, 186403 (2011).
[14] D. I. Khomskii and T. Mizokawa, Orbitally Induced Peierls State in Spinels, Phys. Rev. Lett. 94, 156402 (2005).

[15] D. Pesin and L. Balents, Mott physics and band topology in materials with strong spin-orbit interaction, Nat. Phys. 6, 376 (2010).

[16] A. Go, W. Witczak-Krempa, G. S. Jeon, K. Park, and Y. B. Kim, Correlation Effects on 3D Topological Phases: From Bulk to Boundary, Phys. Rev. Lett. 109, 066401 (2012).

[17] W. Witczak-Krempa and Y. B. Kim, Topological and magnetic phases of interacting electrons in the pyrochlore iridates, Phys. Rev. B 85, 045124 (2012).

[18] X. Wan, A. M. Turner, A. Vishwanath, and S. Y. Savrasov, Topological semimetal and Fermi-arc surface states in the electronic structure of pyrochlore iridates, Phys. Rev. B 83, 205101 (2011).

[19] J. J. Ishikawa, E. C. T. O'Farrell, and S. Nakatsuji, Continuous transition between antiferromagnetic insulator and paramagnetic metal in the pyrochlore iridate $\mathrm{Eu}_{2} \mathrm{Ir}_{2} \mathrm{O}_{7}$, Phys. Rev. B 85, 245109 (2012).

[20] K. Ueda, J. Fujioka, Y. Takahashi, T. Suzuki, S. Ishiwata, Y. Taguchi, and Y. Tokura, Variation of Charge Dynamics in the Course of Metal-Insulator Transition for Pyrochlore-Type $\mathrm{Nd}_{2} \mathrm{Ir}_{2} \mathrm{O}_{7}$, Phys. Rev. Lett. 109, 136402 (2012).

[21] M. C. Shapiro, S. C. Riggs, M. B. Stone, C. R. de la Cruz, S. Chi, A. A. Podlesnyak, and I. R. Fisher, Structure and magnetic properties of the pyrochlore iridate $\mathrm{Y}_{2} \mathrm{Ir}_{2} \mathrm{O}_{7}$, Phys. Rev. B 85, 214434 (2012).

[22] S. M. Disseler, C. Dhital, A. Amato, S. R. Giblin, C. de la Cruz, S. D. Wilson, and M. J. Graf, Magnetic order in the pyrochlore iridates $A_{2} \mathrm{Ir}_{2} \mathrm{O}_{7}(A=\mathrm{Y}, \mathrm{Yb})$, Phys. Rev. B 86, 014428 (2012).

[23] F. F. Tafti, J. J. Ishikawa, A. McCollam, S. Nakatsuji, and S. R. Julian, Pressure-tuned insulator to metal transition in $\mathrm{Eu}_{2} \mathrm{Ir}_{2} \mathrm{O}_{7}$, Phys. Rev. B 85, 205104 (2012).

[24] H. Sagayama, D. Uematsu, T. Arima, K. Sugimoto, J. J Ishikawa, E. O'Farrell, and S. Nakatsuji, Determination of longrange all-in-all-out ordering of $\mathrm{Ir}^{4+}$ moments in a pyrochlore iridate $\mathrm{Eu}_{2} \mathrm{Ir}_{2} \mathrm{O}_{7}$ by resonant X-ray diffraction, Phys. Rev. B 87, 100403(R) (2013).

[25] C. Donnerer, M. C. Rahn, M. M. Sala, J. G. Vale, D. Pincini, J. Strempfer, M. Krisch, D. Prabhakaran, A. T. Boothroyd, and D. F. McMorrow, All-in-all-Out Magnetic Order and Propa- 
gating Spin Waves in $\mathrm{Sm}_{2} \mathrm{Ir}_{2} \mathrm{O}_{7}$, Phys. Rev. Lett. 117, 037201 (2016).

[26] K. Ueda, J. Fujioka, B.-J. Yang, J. Shiogai, A. Tsukazaki, S. Nakamura, S. Awaji, N. Nagaosa, and Y. Tokura, Magnetic Field-Induced Insulator-Semimetal Transition in a Pyrochlore $\mathrm{Nd}_{2} \mathrm{Ir}_{2} \mathrm{O}_{7}$, Phys. Rev. Lett. 115, 056402 (2015).

[27] S. M. Disseler, Direct evidence for the all-in/all-out magnetic structure in the pyrochlore iridates from muon spin relaxation, Phys. Rev. B 89, 140413(R) (2014).

[28] L. Hozoi, H. Gretarsson, J. P. Clancy, B.-G. Jeon, B. Lee, K. H. Kim, V. Yushankhai, P. Fulde, D. Casa, T. Gog, J. Kim, A. H. Said, M. H. Upton, Y.-J. Kim, and J. van den Brink, Longer-range lattice anisotropy strongly competing with spin-orbit interactions in pyrochlore iridates, Phys. Rev. B 89, 115111 (2014).

[29] M. Nakayama, T. Kondo, Z. Tian, J. J. Ishikawa, M. Halim, C. Bareille, W. Malaeb, K. Kuroda, T. Tomita, S. Ideta, K. Tanaka, M. Matsunami, S. Kimura, N. Inami, K. Ono, H. Kumigashira, L. Balents, S. Nakatsuji, and S. Shin, Slater to Mott Crossover in the Metal to Insulator Transition of $\mathrm{Nd}_{2} \mathrm{Ir}_{2} \mathrm{O}_{7}$, Phys. Rev. Lett. 117, 056403 (2016).

[30] H. Guo, K. Matsuhira, I. Kawasaki, M. Wakeshima, Y. Hinatsu, I. Watanabe, and Z.-a. Xu, Magnetic order in the pyrochlore iridate $\mathrm{Nd}_{2} \mathrm{Ir}_{2} \mathrm{O}_{7}$ probed by muon spin relaxation, Phys. Rev. B 88, 060411(R) (2013).

[31] K. Ueda, J. Fujioka, Y. Takahashi, T. Suzuki, S. Ishiwata, Y. Taguchi, M. Kawasaki, and Y. Tokura, Anomalous domainwall conductance in pyrochlore-type $\mathrm{Nd}_{2} \mathrm{Ir}_{2} \mathrm{O}_{7}$ on the verge of the metal-insulator transition, Phys. Rev. B 89, 075127 (2014).

[32] E. Lefrançois, V. Simonet, R. Ballou, E. Lhotel, A. HadjAzzem, S. Kodjikian, P. Lejay, P. Manuel, D. Khalyavin, and L. C. Chapon, Anisotropy-Tuned Magnetic Order in Pyrochlore Iridates, Phys. Rev. Lett. 114, 247202 (2015).

[33] S. M. Disseler, S. R. Giblin, C. Dhital, K. C. Lukas, S. D. Wilson, and M. J. Graf, Magnetization and Hall effect studies on the pyrochlore iridate $\mathrm{Nd}_{2} \mathrm{Ir}_{2} \mathrm{O}_{7}$, Phys. Rev. B 87, 060403(R) (2013).

[34] H. Takatsu, K. Watanabe, K. Goto, and H. Kadowaki, Comparative study of low-temperature $\mathrm{x}$-ray diffraction experiments on $R_{2} \mathrm{Ir}_{2} \mathrm{O}_{7}(R=\mathrm{Nd}, \mathrm{Eu}$, and Pr), Phys. Rev. B 90, 235110 (2014).

[35] S. Zhao, J. M. Mackie, D. E. MacLaughlin, O. O. Bernal, J. J. Ishikawa, Y. Ohta, and S. Nakatsuji, Magnetic transition, longrange order, and moment fluctuations in the pyrochlore iridate $\mathrm{Eu}_{2} \mathrm{Ir}_{2} \mathrm{O}_{7}$, Phys. Rev. B 83, 180402(R) (2011).

[36] H. Guo, C. Ritter, and A. C. Komarek, Direct determination of the spin structure of $\mathrm{Nd}_{2} \mathrm{Ir}_{2} \mathrm{O}_{7}$ by means of neutron diffraction, Phys. Rev. B 94, 161102(R) (2016).

[37] D. Uematsu, H. Sagayama, T.-h. Arima, J. J. Ishikawa, S. Nakatsuji, H. Takagi, M. Yoshida, J. Mizuki, and K. Ishii, Large trigonal-field effect on spin-orbit coupled states in a pyrochlore iridate, Phys. Rev. B 92, 094405 (2015).

[38] W. C. Yang, W. K. Zhu, H. D. Zhou, L. Ling, E. S. Choi, M. Lee, Y. Losovyj, C.-K. Lu, and S. X. Zhang, Robust pinning of magnetic moments in pyrochlore iridates, Phys. Rev. B 96, 094437 (2017).

[39] T. C. Fujita, Y. Kozuka, J. Matsuno, M. Uchida, A. Tsukazaki, T. Arima, and M. Kawasaki, All-in-all-out magnetic domain inversion in $\mathrm{Tb}_{2} \mathrm{Ir}_{2} \mathrm{O}_{7}$ with molecular fields antiparallel to external fields, Phys. Rev. Materials 2, 011402 (2018).
[40] K. Wang, B. Xu, C. W. Rischau, N. Bachar, B. Michon, J. Teyssier, Y. Qiu, T. Ohtsuki, B. Cheng, N. P. Armitage, S. Nakatsuji, and D. van der Marel, Unconventional free charge in the correlated semimetal $\mathrm{Nd}_{2} \mathrm{Ir}_{2} \mathrm{O}_{7}$, Nat. Phys. (2020).

[41] A. Krajewska, T. Takayama, R. Dinnebier, A. Yaresko, K. Ishii, M. Isobe, and H. Takagi, Almost pure $J_{\text {eff }}=\frac{1}{2}$ Mott state of $\mathrm{In}_{2} \mathrm{Ir}_{2} \mathrm{O}_{7}$ in the limit of reduced intersite hopping, Phys. Rev. B 101, 121101(R) (2020).

[42] H. Guo, C. Ritter, and A. C. Komarek, Magnetic structure of $\mathrm{Tb}_{2} \mathrm{Ir}_{2} \mathrm{O}_{7}$ determined by powder neutron diffraction, Phys. Rev. B 96, 144415 (2017).

[43] S. Nakatsuji, Y. Machida, Y. Maeno, T. Tayama, T. Sakakibara, J. van Duijn, L. Balicas, J. N. Millican, R. T. Macaluso, and J. Y. Chan, Metallic Spin-Liquid Behavior of the Geometrically Frustrated Kondo Lattice $\operatorname{Pr}_{2} \operatorname{Ir}_{2} \mathrm{O}_{7}$, Phys. Rev. Lett. 96, 087204 (2006).

[44] Y. Machida, S. Nakatsuji, S. Onoda, T. Tayama, and T. Sakakibara, Time-reversal symmetry breaking and spontaneous Hall effect without magnetic dipole order, Nature (London) 463, 210 (2010).

[45] T. Kondo, M. Nakayama, R. Chen, J. J. Ishikawa, E. G. Moon, T. Yamamoto, Y. Ota, W. Malaeb, H. Kanai, Y. Nakashima, Y. Ishida, R. Yoshida, H. Yamamoto, M. Matsunami, S. Kimura, N. Inami, K. Ono, H. Kumigashira, S. Nakatsuji, L. Balents et al., Quadratic Fermi node in a 3D strongly correlated semimetal, Nat. Commun. 6, 10042 (2015).

[46] L. Savary, E.-G. Moon, and L. Balents, New Type of Quantum Criticality in the Pyrochlore Iridates, Phys. Rev. X 4, 041027 (2014).

[47] E.-G. Moon, C. Xu, Y. B. Kim, and L. Balents, NonFermi-Liquid and Topological States with Strong Spin-Orbit Coupling, Phys. Rev. Lett. 111, 206401 (2013).

[48] X.-P. Yao and G. Chen, $\operatorname{Pr}_{2} \operatorname{Ir}_{2} \mathrm{O}_{7}$ : When Luttinger Semimetal Meets Melko-Hertog-Gingras Spin Ice State, Phys. Rev. X 8, 041039 (2018).

[49] G. Chen, "Magnetic monopole" condensation of the pyrochlore ice U(1) quantum spin liquid: Application to $\operatorname{Pr}_{2} \operatorname{Ir}_{2} \mathrm{O}_{7}$ and $\mathrm{Yb}_{2} \mathrm{Ti}_{2} \mathrm{O}_{7}$, Phys. Rev. B 94, 205107 (2016)

[50] T. Ohtsuki, Z. Tian, A. Endo, M. Halim, S. Katsumoto, Y. Kohama, K. Kindo, S. Nakatsuji, and M. Lippmaa, Spontaneous Hall effect induced by strain in $\operatorname{Pr}_{2} \mathrm{Ir}_{2} \mathrm{O}_{7}$ epitaxial thin films, arXiv:1711.07813.

[51] D. E. MacLaughlin, O. O. Bernal, L. Shu, J. Ishikawa, Y. Matsumoto, J.-J. Wen, M. Mourigal, C. Stock, G. Ehlers, C. L. Broholm, Y. Machida, K. Kimura, S. Nakatsuji, Y. Shimura, and T. Sakakibara, Unstable spin-ice order in the stuffed metallic pyrochlore $\operatorname{Pr}_{2+x} \operatorname{Ir}_{2-x} \mathrm{O}_{7-\delta}$, Phys. Rev. B 92, 054432 (2015).

[52] B.-J. Yang and Y. B. Kim, Topological insulators and metalinsulator transition in the pyrochlore iridates, Phys. Rev. B 82, 085111 (2010).

[53] G. Chen and M. Hermele, Magnetic orders and topological phases from $f$ - $d$ exchange in pyrochlore iridates, Phys. Rev. B 86, 235129 (2012).

[54] R. Wang, A. Go, and A. Millis, Weyl rings and enhanced susceptibilities in pyrochlore iridates: $k \cdot p$ analysis of cluster dynamical mean-field theory results, Phys. Rev. B 96, 195158 (2017).

[55] J.-W. Rhim and Y. B. Kim, Quantum oscillations in the Luttinger model with quadratic band touching: Applica- 
tions to pyrochlore iridates, Phys. Rev. B 91, 115124 (2015).

[56] B.-J. Yang and N. Nagaosa, Emergent Topological Phenomena in Thin Films of Pyrochlore Iridates, Phys. Rev. Lett. 112, 246402 (2014).

[57] E. K.-H. Lee, S. Bhattacharjee, and Y. B. Kim, Magnetic excitation spectra in pyrochlore iridates, Phys. Rev. B 87, 214416 (2013).

[58] R. Wang, A. Go, and A. J. Millis, Electron interactions, spinorbit coupling, and intersite correlations in pyrochlore iridates, Phys. Rev. B 95, 045133 (2017).

[59] S. Maekawa, S. E. Barnes, S. Ishihara, G. Khaliullin, W. Koshibae, and T. Tohyama, Physics of Transition Metal Oxides (Springer, New York, 2004).

[60] K. A. Ross, L. Savary, B. D. Gaulin, and L. Balents, Quantum Excitations in Quantum Spin Ice, Phys. Rev. X 1, 021002 (2011).

[61] H. Yan, O. Benton, L. Jaubert, and N. Shannon, Theory of multiple-phase competition in pyrochlore magnets with anisotropic exchange with application to $\mathrm{Yb}_{2} \mathrm{Ti}_{2} \mathrm{O}_{7}, \mathrm{Er}_{2} \mathrm{Ti}_{2} \mathrm{O}_{7}$, and $\mathrm{Er}_{2} \mathrm{Sn}_{2} \mathrm{O}_{7}$, Phys. Rev. B 95, 094422 (2017).

[62] M. Elhajal, B. Canals, R. Sunyer, and C. Lacroix, Ordering in the pyrochlore antiferromagnet due to Dzyaloshinsky-Moriya interactions, Phys. Rev. B 71, 094420 (2005).

[63] L. Savary, K. A. Ross, B. D. Gaulin, J. P. C. Ruff, and L. Balents, Order by Quantum Disorder in $\mathrm{Er}_{2} \mathrm{Ti}_{2} \mathrm{O}_{7}$, Phys. Rev. Lett. 109, 167201 (2012).

[64] C. Zener, Interaction between the $d$-Shells in the Transition Metals. II. Ferromagnetic Compounds of Manganese with Perovskite Structure, Phys. Rev. 82, 403 (1951).

[65] R. Okuma, D. Ueta, S. Kuniyoshi, Y. Fujisawa, B. Smith, C. H. Hsu, Y. Inagaki, W. Si, T. Kawae, H. Lin, F. C. Chuang,
T. Masuda, R. Kobayashi, and Y. Okada, Fermionic order by disorder in a van der Waals antiferromagnet, arXiv:2007.15193 [cond-mat.str-el].

[66] X. Zhou, H. Li, J. A. Waugh, S. Parham, H.-S. Kim, J. A Sears, A. Gomes, H.-Y. Kee, Y.-J. Kim, and D. S. Dessau, Angle-resolved photoemission study of the Kitaev candidate $\alpha-\mathrm{RuCl}_{3}$, Phys. Rev. B 94, 161106(R) (2016).

[67] K. W. Plumb, J. P. Clancy, L. J. Sandilands, V. V. Shankar, Y. F. $\mathrm{Hu}, \mathrm{K} . \mathrm{S}$. Burch, H.-Y. Kee, and Y.-J. Kim, $\alpha-\mathrm{RuCl}_{3}$ : A spinorbit assisted Mott insulator on a honeycomb lattice, Phys. Rev. B 90, 041112(R) (2014).

[68] L. J. Sandilands, Y. Tian, A. A. Reijnders, H.-S. Kim, K. W. Plumb, Y.-J. Kim, H.-Y. Kee, and K. S. Burch, Spin-orbit excitations and electronic structure of the putative Kitaev magnet $\alpha-\mathrm{RuCl}_{3}$, Phys. Rev. B 93, 075144 (2016).

[69] A. Koitzsch, C. Habenicht, E. Müller, M. Knupfer, B. Büchner, H. C. Kandpal, J. van den Brink, D. Nowak, A. Isaeva, and Th. Doert, $J_{\text {eff }}$ Description of the Honeycomb Mott Insulator $\alpha-\mathrm{RuCl}_{3}$, Phys. Rev. Lett. 117, 126403 (2016).

[70] P. Ong, lecture delivered at Princeton Summer School on Condensed Matter Physics, 2020 (unpublished).

[71] T. Yokoi, S. Ma, Y. Kasahara, S. Kasahara, T. Shibauchi, N. Kurita, H. Tanaka, J. Nasu, Y. Motome, C. Hickey, S. Trebst, and Y. Matsuda, Half-integer quantized anomalous thermal Hall effect in the Kitaev material $\alpha-\mathrm{RuCl}_{3}$, arXiv:2001.01899 [condmat.str-el].

[72] Y. Kasahara, K. Sugii, T. Ohnishi, M. Shimozawa, M. Yamashita, N. Kurita, H. Tanaka, J. Nasu, Y. Motome, T. Shibauchi, and Y. Matsuda, Unusual Thermal Hall Effect in a Kitaev Spin Liquid Candidate $\alpha-\mathrm{RuCl}_{3}$, Phys. Rev. Lett. 120, 217205 (2018). 